Revue Française de Civilisation Britannique

XIV-1 | 2006

La dévolution des pouvoirs à l'Écosse et au Pays de Galles 1966-1999

\title{
La dévolution au miroir de l'histoire impériale
}

Devolution through the glass of imperial history

Antoine Mioche

\section{OpenEdition}

1 Journals

Édition électronique

URL : http://journals.openedition.org/rfcb/1181

DOI : $10.4000 /$ rfcb. 1181

ISSN : 2429-4373

Éditeur

CRECIB - Centre de recherche et d'études en civilisation britannique

Édition imprimée

Date de publication : 2 janvier 2006

Pagination : 177-191

ISBN : 2-911580-23-0

ISSN : 0248-9015

Référence électronique

Antoine Mioche, "La dévolution au miroir de l'histoire impériale », Revue Française de Civilisation

Britannique [En ligne], XIV-1 | 2006, mis en ligne le 15 octobre 2016, consulté le 27 avril 2019. URL:

http://journals.openedition.org/rfcb/1181; DOI : 10.4000/rfcb.1181

\section{(c) (i) $\odot$}

Revue française de civilisation britannique est mis à disposition selon les termes de la licence Creative Commons Attribution - Pas d'Utilisation Commerciale - Pas de Modification 4.0 International. 


\title{
La dévolution au miroir de l'histoire impériale
}

\author{
Antoine MIOCHE \\ Université de Paris III — Sorbonne Nouvelle
}

L'Empire aurait-il préfiguré l'heureuse co-existence et l'unité, dans la souveraineté parlementaire de Westminster, de multiples communautés autogouvernées, tantôt unies entre elles, tantôt non ? Peut-on parler dans leur cas de dévolution, comme semble le faire un des meilleurs spécialistes britanniques de la question ? ${ }^{1}$ Dans quelle mesure peut-on, à propos de la dévolution, raisonner sur la métropole par analogie avec l'Empire?

\section{Une fausse antinomie}

Le Royaume-Uni est un État dont la cohésion des parties — deux d'entre elles, l'Écosse et le pays de Galles prétendant à une dignité nationale égale à celle de l'Angleterre, et l'Irlande du nord divisée entre allégeances rivales - est assurée par la Couronne-en-son-Parlement. La souveraineté du Parlement de Westminster scelle l'unité d'une entité composite, au sein de laquelle les vicissitudes de l'histoire ont laissé subsister, selon des modalités et à des degrés divers, des traits nationaux antérieurs à l'union de l'Angleterre et de ses périphéries. Quoique la souveraineté n'y soit pas divisée ou répartie entre plusieurs niveaux de gouvernement, il présente donc la spécificité de dessiner un espace où le débat sur le degré d'uniformité imposé à une structure multinationale complexe par l'appareil d'un État unique est de l'essence même de cet État. Pour le dire autrement, l'Union n'est pas une structure constitutionnelle statique, mais bien plutôt un cadre de négociation de la relation entre l'Angleterre, qui la voulut d'abord, et ses marges. Certes centralisé, le Royaume-Uni institue ainsi, et interroge tout à la fois, un équilibre dynamique quelquefois rompu, comme dans le cas de l'Irlande — entre unité et diversité.

Le processus de dévolution entamé par le gouvernement Blair élu en 1997 apparaît, de ce point de vue, parfaitement conforme à l'essence de l'Union, par sa nature de tentative d'articuler à nouveaux frais l'équilibre constitutionnel qui la fonde et lui donne sens. Et pour cette raison, il est sans doute plus juste de considérer que c'est le risque, avéré ou seulement perçu, de désunion du royaume, qui a produit la dévolution, plutôt que l'inverse. Si néanmoins la question peut légitimement se poser de la compatibilité entre dévolution et unité du royaume, il faut donc en chercher la raison ailleurs que dans une opposition antinomique : dans cette relation négociée entre l'Angleterre et sa périphérie qui met en jeu le principe

\footnotetext{
${ }^{1}$ Vernon BOGDANOR, Devolution in the United Kingdom, Oxford: OUP, 1979 (second edition 2001), p. 23.
} 
central de la constitution et de la culture politique du Royaume-Uni depuis le tournant des XVII ${ }^{\mathrm{e}}$ et XVIII ${ }^{\mathrm{e}}$ siècles, la souveraineté du Parlement de Londres.

\section{La souveraineté parlementaire}

De fait, ce sont les réticences à laisser subsister deux parlements au sein d'une union dynastique qui poussèrent, côté anglais, à rechercher l'union législative. Il ne fallait pas que l'alliance nouée lors de la Glorieuse Révolution entre Couronne et Parlement anglais pût se défaire par l'appui qu'un parlement étranger apporterait à un monarque mal intentionné - danger singulièrement réel au tournant du XVIII ${ }^{\mathrm{e}}$ siècle, si peu de temps après que les Stuart catholiques ont été chassés du trône, auquel ils continuent de prétendre. Par comparaison, l'« union » anglo-galloise de 1536-43 fut une pure incorporation, certes à des fins similaires (il fallait que le pouvoir du monarque anglais comme chef de l'Église anglicane s'étendît au pays de Galles en même temps que son pouvoir temporel de seigneur féodal, à défaut de quoi la coïncidence anglaise entre Église et État, nouée par la réforme henricienne, serait imparfaite), mais selon une technique différente. Et par comparaison, toujours, l'Irlande fut d'abord gouvernée séparément et, dès les dernières années du XV $\mathrm{XV}^{\mathrm{e}}$ siècle et jusqu'en 1782-83, rigoureusement subordonnée à l'Angleterre par des artifices législatifs et politiques, sans recours à une union législative. Ainsi, c'est bien l'union constitutive du Royaume-Uni de Grande-Bretagne en 1707 qui a pour ressort intime la souveraineté parlementaire, absente du tableau dans le pays de Galles du $\mathrm{XVI}^{\mathrm{e}}$ siècle, et simple instrument de contrôle de l'Irlande à la fin du $\mathrm{XVIII}^{\mathrm{e}}$ siècle, devant l'insuffisance des modalités antérieures de maintien sous tutelle. Elle apparaît à ce titre comme une innovation par rapport à l'absorption du pays de Galles, et comme clairement distincte du cas très original de l'Irlande, royaume doté d'un parlement, certes, mais subordonné.

Le cas de l'Irlande est utile ici pour entrer profitablement dans la discussion de la dévolution, non seulement parce que le débat sur la dévolution dans sa forme contemporaine y prendra naissance au $\mathrm{XIX}^{\mathrm{e}}$ siècle, mais parce que l'Irlande illustre elle aussi une transition importante : de la non-incorporation à l'incorporation, par le passage du gouvernement représentatif subordonné (à la Couronne anglaise, puis au Parlement britannique) à l'union législative. Bien avant d'être au début des années 1920, dans sa partie septentrionale, la première région du Royaume-Uni à faire (contre son inclination) l'expérience de la dévolution, l'Irlande est en effet aussi la première et la seule région de l'espace britannique à passer d'un statut de subordination externe (l'Écosse, par contraste, était un royaume indépendant) à une position d'inclusion. Car la norme, dans cet espace, comme on l'oublie trop volontiers, et comme l'histoire constitutionnelle de l'Empire vient nous le rappeler, ce n'est pas l'union législative.

\section{L'union, phénomène atypique}

Ainsi, jamais les colonies, quelles qu'elles soient, ne seront représentées en tant que telles à Westminster. En revanche, les colonies de peuplement se verront très tôt dotées d'assemblées législatives, même si là aussi les choses se firent à des époques, dans des circonstances, selon des formes, et avec des fortunes, variables. La Jamaïque, 
notamment, possède la sienne dès le XVII ${ }^{\mathrm{e}}$ siècle, tout comme un certain nombre des colonies du continent américain, et le Québec s'en voit octroyer une en 1774, le Nouveau-Brunswick en 1785 . Au XVII ${ }^{\mathrm{e}}$ siècle, la tendance des colonies à s'affranchir, par calcul ou par ignorance du droit, de l'autorité monarchique au moyen de ces assemblées crée déjà des tensions entre Londres et les colonies d'Amérique continentale, et suscite sous Jacques II des efforts considérables de reprise en mains, de rationalisation et de centralisation de l'administration impériale. Au XVIII ${ }^{\mathrm{e}}$ siècle, la même attitude tend à remettre en cause, non plus seulement l'autorité du monarque, mais celle aussi de son Parlement, sans que la solution écossaise ou irlandaise d'une incorporation législative s'offre pour remédier au problème. Pendant longtemps, le vrai défi du monde britannique, sous le rapport constitutionnel, ne sera donc pas de préserver l'union métropolitaine, mais de gouverner hors de ses frontières sans pouvoir l'étendre.

L'Union, ou si l'on préfère les trois unions, de métropole ont vocation à assurer une fonction bien spécifique, la cohérence de l'Angleterre avec elle-même, son intégrité constitutionnelle, qui se résume d'abord en une coïncidence de l'Église, de l'État et de la nation, à laquelle se surajoute ensuite l'exercice conjoint de la souveraineté par le monarque et le Parlement. Elles y sont nécessaires et elles y suffisent. Elles ne sont pas l'instrument d'un projet d'expansion, mais la réaction de l'Angleterre à une situation de vulnérabilité. Cependant, après la Glorieuse Révolution, la Grande-Bretagne hérite du gouvernement d'un Empire où les assemblées législatives coloniales sont nombreuses. C'est ce qui explique que l'on puisse voir aujourd'hui dans cette rémanence de l'époque antérieure à 1707, hors des frontières métropolitaines, sinon peut-être un modèle de dévolution avant l'heure, du moins un point de comparaison fécond avec la situation présente.

\section{Union et Empire}

Que la dévolution ait d'abord fait question dans le contexte des relations entre le Royaume-Uni et l'Irlande du XIX ${ }^{\mathrm{e}}$ siècle, plutôt que dans l'Écosse du siècle précédent, n'est pas fortuit et se révèle d'un intérêt non négligeable pour mesurer la validité de l'analogie entre Union et Empire. L'hostilité du peuple écossais à l'Union, en effet, s'exprime dans la première moitié du XVIII ${ }^{\mathrm{e}}$ siècle par le biais du jacobitisme, c'est-à-dire par le soutien à la branche de la dynastie Stuart chassée du trône à la Glorieuse Révolution, et l'espoir de renverser l'union législative afin d'en revenir à la formule antérieure d'une union strictement monarchique. Il ne s'agit pas ici d'aménager l'Union, mais de revenir à l'état précédent. En revanche, la dévolution en Irlande, apparue sous la forme longtemps imprécise du Home Rule, se veut d'entrée un compromis entre l'indépendance et une union dont l'un des défauts majeurs est de perpétuer l'infériorité politique de la majorité de la population. La dévolution, dans ce contexte irlandais, se fait jour comme une affaire, non simplement d'autonomie - question à l'ordre du jour dans les colonies de peuplement — mais d'autonomie dans le sein de l'Union.

Ainsi se heurte-t-on très vite à une des limites de la comparaison entre Union et Empire. L'Union, c'est ce qui est nécessaire et suffisant à la préservation de l'« empire » (c'est-à-dire l'autorité souveraine) de l'Angleterre sur elle-même. C'est pourquoi il ne saurait y avoir d'admission à l'Union de la part d'un territoire extra-métropolitain qui en remette en cause le fondement - cas des colonies continentales d'Amérique dans les 
années 1760. C'est pourquoi, de même, un territoire admis à l'Union, mais menaçant son intégrité, peut et doit en dernier ressort en être exclus — cas de l'Irlande du sud en 1921. C'est ce minimalisme qui distingue l'Union du reste de l'Empire britannique. Il y a, certes, continuité de l'une à l'autre après 1688-89 par le truchement théorique de la souveraineté de Westminster. Et, certes, cette doctrine de la souveraineté parlementaire outre-mer a pour fonction d'empêcher la dissociation hors du Royaume-Uni de l'alliance entre autorité royale et autorité parlementaire qu'a instituée la révolution de 1688-89 c'est-à-dire, de manière en un sens lointaine, de préserver encore le cœur anglais de l'Empire. Mais cette souveraineté du Parlement impérial s'exerce en l'absence de toute représentation coloniale directe, précisément parce que le maintien de l'«empire» de l'Angleterre sur elle-même ne saurait s'accommoder d'une représentation coloniale à Westminster ${ }^{2}$. Comme un commentateur devait l'écrire en 1859, le Parlement britannique était «impérial par l'étendue de son pouvoir, et non en vertu de sa composition $»^{3}$. En ce sens, l'Empire britannique est l'opposé, ou du moins le complément, de l'Union. Il commence (comme notion) là où l'Union (comme pratique) s'arrête.

Est-ce à dire que la question de l'articulation de l'Union à l'Empire ne porte aucun enseignement pour la dévolution ? Non, bien sûr, puisque l'on voit dans les deux sphères se poser la question de la compatibilité de la souveraineté du Parlement de Westminster avec l'exercice local du gouvernement par assemblée ou parlement. Mais il doit être bien clair que la dévolution est une notion qui n'a de sens que dans le sein géographique et constitutionnel de l'Union. Ce que l'on observera au dehors pourra suggérer des parallèles (encore faut-il ne jamais perdre de vue que les assemblées coloniales pré-existent à l'émergence de la doctrine de la souveraineté parlementaire de Westminster), on n'en aura pas pour autant dévolution, puisque l'Empire constitue en soi, comme l'Union, mais séparément d'elle, une réponse spécifique à la question première et dernière de la préservation de l'intégrité du noyau anglais. Dans le domaine de la juridiction de la Couronne et de son parlement, il est ce qui ne saurait rentrer dans l'Union et pose par voie de conséquence la question de son articulation à la métropole.

Dès lors que la souveraineté devient monarcho-parlementaire, on peut en effet se demander si l'autorité du parlement s'étend aux colonies, si elle y suit en quelque sorte l'autorité royale - une réponse négative à cette interrogation ayant pour effet possible de désolidariser la Couronne et le parlement, et par voie de conséquence, de menacer de rouvrir en métropole la querelle de la succession (tant que le pouvoir des Hanovre n'est pas stabilisé, ce qui ne se fera qu'à partir de la répression du dernier soulèvement jacobite en 1746), mais aussi les conflits civils du XVII ${ }^{\mathrm{e}}$ siècle autour de la prérogative royale (renforcée de surcroît par l'existence outre-mer de vastes et riches contrées sous sa seule autorité) et de la liberté religieuse. L'Empire soulève donc à deux titres la question de l'unité - en tant que la périphérie peut ou non être intégrée au centre, et en tant qu'elle a la capacité, dans ce cadre de réflexion, de

${ }^{2}$ Certains « coloniaux » siégeront à Westminster, mais ils seront élus par des circonscriptions métropolitaines.

${ }^{3}$ T. C. M. MEEKINS, Parliamentary Reform, Should the Colonies be Represented? London, 1859 , p. 4, cité par Ged MARTIN, 'Empire federalism and imperial parliamentary union, 1820-1870’, Historical Journal, vol. XVI, n I (1973), pp. 65-92 (p. 74). 
remettre en cause l'unité du centre lui-même. C'est ce que révèle la crise américaine des années 1760-70, inaugurée par la controverse du Stamp Act.

\section{Maintenir l'unité de la Couronne et du Parlement}

La dispute, déclenchée par une mesure fiscale votée par le Parlement de Westminster et contestée par les Américains, porte en dernier ressort sur la question de savoir si le lien d'union avec la métropole se fait par la législature impériale (où les colons ne sont pas représentés) ou par la Couronne et la common law ${ }^{4}$. Trois options se dessinent: le maintien des assemblées coloniales, assorti néanmoins d'une représentation à Westminster pour faire droit au principe de consentement à la législation du Parlement impérial, reconnu comme souverain ; le statu quo ; et l'attache par la seule monarchie, qui signifierait le déni de la souveraineté du Parlement de Londres.

Nombreux sont ainsi, dans les années 1760-70, les projets de représentation américaine à Westminster. Ils font varier le nombre, la qualité et la fonction des représentants, mais partagent le double souci de reconnaître la britannicité des coloniaux et de veiller à ce que l'autorité de la législature impériale s'étende dans les mêmes limites que l'autorité royale ${ }^{5}$. Toutefois, l'agglomération à l'union métropolitaine, elle-même si neuve, d'une masse territoriale considérable, contiguë à des territoires sous gouvernement étranger, lointaine et donc difficile à administrer et à défendre, peuplée en majorité de protestants dissidents ${ }^{6}$ et parcourue de divisions religieuses, marquée enfin par l'institution de l'esclavage, et menaçant de surcroît de faire basculer le centre de gravité démographique, économique et politique de l'Empire, comme l'avait déjà dit Benjamin Franklin dans les années $1750^{7}$, ne saurait se concevoir qu'avec peine du côté métropolitain. Elle aurait en outre pour corollaire, selon l'analogie écossaise, la suppression des assemblées coloniales. Il n'est donc pas possible de concevoir une représentation coloniale à

\footnotetext{
${ }^{4}$ Voir J.C.D. CLARK, The Language of Liberty 1660-1832. Political Discourse and Social Dynamics in the Anglo-American world, Cambridge: Cambridge University Press, 1994, chapitre 1.

${ }^{5}$ Charles F. MULLETT note, in : 'English Imperial Thinking', Political Science Quarterly vol. XLV, Number 4 (December 1930), pp. 548-579, que la théorie de la dépendance des colonies vis-à-vis de la seule Couronne a cours dès 1759 (p. 552, n. 15). Elle est suffisamment établie en 1768 pour que Thomas POWNALL s'emploie à la réfuter dans la quatrième édition de son Administration of the Colonies, et à proposer au contraire une représentation américaine à Westminster pour pallier le risque d'une sécession américaine ; voir G. H. GUTTRIDGE, 'Thomas Pownall's The Administration of the Colonies: The Six Editions', William and Mary Quarterly, vol. XXVI, N 1 (1969), pp. 31-46, (pp. 39-41).

${ }^{6}$ Trois quarts des quelque deux millions de colons américains sont des protestants non anglicans. Selon Edmund BURKE, Speech of Edmund Burke, Esq. on Moving his Resolutions for Conciliation with the Colonies, March 22, 1775, $3^{\mathrm{e}}$ éd., London: printed for J. Dodsley, 1775 , p. 32, un dixième seulement des colons étaient anglicans.

${ }^{7}$ Benjamin FRANKLIN, 'Observations concerning the increase of mankind, peopling of countries, etc.' (1751), in : Albert Henry SMYTH (ed.), The Writings of Benjamin Franklin Collected and Edited with a Life and Introduction, 10 vol., Vol. III, 1750-1759, New York : The Macmillan Company, 1905, pp. 63-73, en particulier p. 71. La population américaine, estime Franklin, double tous les vingt-cinq ans par l'effet du seul accroissement naturel.
} 
Londres qui réponde au modèle de 1707, car il n'est pas reproductible, malgré son caractère de référence. C'est pourquoi ces projets d'union restent lettre morte.

On voit en revanche réaffirmer la souveraineté de Westminster, sans reconnaître aux colons le droit de se taxer. C'est l'argument, stérile dans les circonstances d'une crise déclarée, du statu quo, celui de la représentation virtuelle. Dès lors qu'une fraction à peine de la population britannique participe à l'élection de députés, objecte-t-on aux Américains, l'argument de l'inapplicabilité et de l'illégitimité d'une loi faute de participation de ses justiciables à l'élection de ceux qui, par le vote, expriment l'assentiment de la nation, ne saurait s'entendre absolument. Les représentants de la nation à Westminster sont ceux de la nation entière, coloniaux compris, et non seulement des électeurs. Accepter la théorie américaine de l'illégitimité de l'autorité du Parlement impérial pouvait, selon Josiah Tucker, avoir des conséquences graves pour l'ordre métropolitain :

puisque dix-neuf personnes sur vingt en Angleterre, et plus de quatrevingt-dix-neuf sur cent en Écosse, n'ont pas la qualification requise pour être électeurs, et ne l'ont jamais été, si considérables qu'aient pu être leurs biens, c'est-à-dire (selon cette nouvelle doctrine) ne sont pas représentées au Parlement; il s'ensuit inévitablement que l'écrasante majorité des habitants de Grande-Bretagne, aussi bien que de l'Amérique britannique, ont le droit de renoncer à leur allégeance au présent gouvernement dès qu'il leur plaira et de se rendre indépendants. ${ }^{8}$

À défaut qu'elles reconnaissent la souveraineté de Westminster, Tucker préférait en conséquence l'abandon pur et simple des treize colonies, c'est-à-dire un repli qui permît, là encore, de continuer à assurer la coïncidence entre l'autorité de la Couronne et celle du Parlement.

\section{Burke, dévolutionniste?}

Edmund Burke, pour sa part, devait proposer de maintenir cette coïncidence en réaffirmant cette souveraineté, mais en suggérant qu'elle ne fût pas exercée. Dans son célèbre discours de conciliation des colonies américaines du 22 mars 1775, au terme de dix années de polémique alimentée de part et d'autre de l'Atlantique par des centaines de livres et de pamphlets, il dresse le constat qui s'imposait de l'impossibilité et de l'inopportunité de l'incorporation des colonies américaines au Royaume-Uni ${ }^{9}$. De ce constat, toutefois, il ne tire pas que les colonies étaient

\footnotetext{
${ }^{8}$ Josiah TUCKER, An Humble Address and Earnest Appeal to those Respectable Personages in Great-Britain and Ireland, Who, by their Great and Permanent Interest in Landed Property, their Liberal Education, Elevated Rank, and Enlarged Views, are the Ablest to Judge, and the Fittest to Decide, Whether a Connection with, or a Separation from the Continental Colonies of America, be Most for the National Advantage, and the Lasting Benefit of these Kingdoms, Glocester [sic] : printed by R. Raikes and sold by T. Cadell, 1775, p. 87.

${ }^{9}$ Speech of Edmund Burke, op. cit. À la même époque, Smith croit encore à la possibilité de l'union, mais en 1778, il concèdera que l'opinion publique britannique est plus favorable à
} 
appelées à prendre leur indépendance. Il est au contraire d'avis que la paix, la concorde, l'unité et la prospérité de l'Empire britannique seraient assurées par la reconnaissance aux assemblées coloniales du droit de voter librement en Amérique leur contribution aux dépenses publiques imputables en Amérique. C'est ce qu'en retient Vernon Bogdanor.

Cet éminent juriste, toutefois, ne souligne pas assez que le modèle de Burke et c'est très parlant — est l'Irlande, qui ne fait alors pas encore partie de l'union métropolitaine. «L'on dit [...], écrit Burke, que ce pouvoir de voter les subventions accordé aux assemblées américaines, dissoudrait l'unité de l'empire [...]. » Mais «ce que signifie cette unité », il l'ignore, car "l'idée même de subordination des parties exclut cette notion d'une unité simple et entière. L'Angleterre est la tête; mais elle n'est pas la tête et les membres aussi. L'Irlande depuis le début a toujours eu une législature séparée, mais non indépendante ", ce qui fut à l'avantage de l'union. Tout était fait pour la préservation de l'autorité de l'Angleterre sur l'Irlande, dans le même temps que les libertés anglaises étaient aussi introduites dans l'île. «Je ne sache pas que l'on ne puisse adopter les mêmes principes dans vingt îles » plutôt qu'une, c'est-à-dire les colonies américaines continentales et insulaires, « et avec le même heureux effet $\gg .^{10}$

Il ne doit donc pas y avoir de malentendu sur le parallèle que fait Burke. Comme il a soin de le souligner, l'Irlande était un royaume subordonné à la suprématie du Parlement britannique. En outre, son discours porte, non point sur la nature exacte du lien constitutionnel, mais sur la nature de l'Empire. Or, l'Empire, pour lui, n'est pas une union incorporatrice. Et c'est pour cela qu'envisager d'accorder le privilège de taxation aux assemblées coloniales n'est au fond pas problématique à ses yeux : ce droit, insiste-t-il, a d'ailleurs pleinement fonctionné lors de la Guerre de Sept Ans, avec la bénédiction et à l'avantage de la métropole ${ }^{11}$. En d'autres termes, le droit colonial à légiférer dans des assemblées localement élues ne peut qu'être reconnu, car il est le seul moyen de réaliser l'unité quand la conquête et l'union législative sont également impossibles. Il y a certes là, si l'on veut, un mécanisme dévolutif, mais — c'est la seule correction qu'il faille apporter au propos de Bogdanor - il a vocation à s'appliquer précisément parce que l'union est impossible, et non pour rendre l'union possible.

En outre, et l'on retrouve ici un trait du débat sur la dévolution au tournant des $\mathrm{XX}^{\mathrm{e}}$ et $\mathrm{XXI}^{\mathrm{e}}$ siècles, ce self-government dans l'Empire recèle, pour ses partisans comme ses détracteurs, le potentiel de faire éclater l'Amérique en une multitude de royaumes, avec ce risque (ou cet avantage, selon le point de vue) que la souveraineté du Parlement de Westminster ne s'étende plus hors de métropole et que, par voie de conséquence, Couronne et Parlement soient disjoints. C'est ce qu'illustre le projet

une victoire militaire. V. Adam SMITH, An Inquiry into the Nature and Causes of the Wealth of Nations (1776), R.H. CAMPBELL \& A.S. SKINNER (eds.), 2 vol., Oxford: Clarendon Press, 1976, Vol. 2, p. 624-25; et G.H. GUTTRIDGE, 'Adam Smith on the American Revolution: an Unpublished Memorial', American Historical Review, vol. XXXVIII, $\mathrm{N}^{\circ} 4$ (July 1933), pp. 711-20 (p. 717).

${ }^{10}$ Speech of Edmund Burke, op. cit., p. 93.

${ }^{11}$ Ibid., p. 78-82. 
du Major John Cartwright ${ }^{12}$. Ici, le précédent impérial serait donc plutôt inquiétant que rassurant pour l'avenir de l'Union.

\section{«Quinze royaumes indépendants »}

Cartwright se fait l'avocat de la séparation des colonies américaines aux deux motifs que «les droits de souveraineté résident dans le peuple lui-même; c'est-àdire qu'il a le droit de choisir lui-même ceux qui vont le gouverner », et que «les Américains sont un peuple libre ». Cette indépendance vis-à-vis du Parlement de Westminster, cependant, ne se confond pas avec une séparation totale. Si Cartwright partage avec Tucker l'idée qu'il convient d'en venir à une sécession, il entend néanmoins qu'elle se fasse au sein d'une nation britannique, certes redéfinie, mais maintenue. Les sujets du roi, explique-t-il à ce propos, ne cessent pas d'être ses sujets en cessant d'être soumis à l'autorité du Parlement britannique : "de même qu'ils étaient sujets en Angleterre [avant l'émigration], de même ils sont sujets dans leur nouveau pays ». Il ne s'agit pas pour Cartwright de "promouvoir l'abandon ingrat de la mère patrie par ses enfants », mais de substituer à une relation de subordination, une relation d'égalité fraternelle: «Je souhaite considérer les gouvernements américains, comme celui de l'Irlande, comme des royaumes sœurs », mais, à la différence de l'Irlande (dont il juge que l'importance stratégique justifie sa réunion à l'île principale voisine), non subordonnés à la Grande-Bretagne par la suprématie de Westminster ${ }^{13}$. Echo significatif d'arguments avancés dès le XVII siècle, à propos de l'Irlande, par des polémistes tels que Patrick Darcy (dans un discours de 1641) et William Molyneux (dans son célèbre plaidoyer The Case of Ireland Being Bound by Acts of the English Parliament), pour faire le départ entre l'autorité de la Couronne et celle du Parlement ${ }^{14}$. Écho aussi de revendications américaines de la même fin du XVII ${ }^{\mathrm{e}}$ siècle $^{15}$.

\footnotetext{
${ }^{12}$ John CARTWRIGHT, American independence the interest and glory of Great Britain; : containing arguments which prove, that not only in taxation, but in trade, manufactures, and government, the colonies are entitled to an entire independency on the British legislature; and that it can only be by a formal declaration of these rights, and forming thereupon a friendly league with them, that the true and lasting welfare of both countries can be promoted. : In a series of letters to the legislature, Philadelphie: Robert Bell, 1776. (Reprise de l'édition anglaise de 1774, publiée à Londres).

${ }^{13}$ Ibid., pp. 35, 36, 37, 41, 55 \& 57.

${ }^{14}$ Sur les positions de Molyneux et Darcy en matière de souveraineté parlementaire, voir Caroline ROBBINS, The Eighteenth-Century Commonwealthman: Studies in the Transmission, Development and Circumstance of English Liberal Thought from the Restoration of Charles II until the War with the Thirteen Colonies, New York: Atheneum, 1968 ( $1^{\text {ère }}$ publication en 1959), pp. 139-140. Cette disjonction - qui entraîne, hors d'Angleterre, une solution de continuité territoriale et constitutionnelle dans l'Empire - leur vaut l'étiquette de « républicains ». Républicanisme qui n'est en vérité, nonobstant la surprise affichée par Caroline Robbins, nullement incompatible avec la monarchie, puisqu'il s'articule dans une prise de position sur la définition de la base confessionnelle et constitutionnelle de l'État et de la nation en Angleterre.

${ }^{15}$ Voir David S. LOVEJOY, 'Two American Revolutions, 1689 and 1776', in: J.G.A. POCOCK (ed.), Three British Revolutions: 1641, 1688, 1776, Princeton, New Jersey: Princeton University Press, 1980, pp. 244-262 (pp. 245-249).
} 
Cartwright fait du principe du consentement le fondement de la sécession des colonies américaines. Il en découle en outre pour lui que la séparation doit se faire entre chaque colonie et la métropole, les colonies étant reconnues comme formant autant d'«États libres et indépendants» dont le monarque britannique serait individuellement le souverain ${ }^{16}$. Ce seraient "quinze royaumes indépendants ${ }^{17}$, placés sous la protection bienveillante du Parlement de Grande-Bretagne selon les termes d'un ensemble d'accords internationaux, et en vertu de relations diplomatiques, qui constitueraient «une ligue» d'aspect confédéral, dominée toutefois par une Grande-Bretagne qui conserverait le «pouvoir de tenir par la peur l'Amérique au respect de ses traités ${ }^{18}$. Cartwright, pourtant défenseur des libertés, se fait ainsi l'avocat paradoxal d'un retour à une position d'éminence de la monarchie, inacceptable tant que la monarchie remplit encore un rôle politique autre que principalement symbolique, aussi bien que de la Grande-Bretagne.

Nonobstant la gravité de la crise américaine, il était d'ailleurs possible (fort heureusement, du point de vue des autorités métropolitaines) de circonvenir ou de manipuler les assemblées coloniales conformément à la logique d'ancien régime où leur fonction n'était pas tant de représenter la population, que d'aider le gouvernement à exercer son autorité. C'est le sens, par exemple, de la création de conseils nommés au Québec en 1774, comme le comprirent fort bien les Américains rétifs. Et c'est une autre raison de ne pas confondre self-government colonial au $\mathrm{XVIII}^{\mathrm{e}}$ siècle et dévolution dans le Royaume-Uni d'aujourd'hui. En revanche, une analogie plus probante est à rechercher, à partir de 1848, dans la concession de gouvernements coloniaux responsables devant leurs assemblées élues. La mesure, qui fait d'abord, très traditionnellement craindre la disjonction entre la Couronne et le Parlement, l'évolution vers ce que Cartwright avait appelé des «royaumes indépendants », apparaît alors comme le gage d'une unité maintenue.

\section{«Une république nouvelle et indépendante »}

Après la paix de Paris de 1763, les autorités métropolitaines furent confrontées, dans la vaste province de Québec gagnée sur les Français, à la nécessité de faire co-habiter ceux que l'on appelait les «nouveaux sujets» ou «Canadiens » - majoritaires et catholiques - avec les «anciens sujets 》minoritaires et protestants - en vérité d'établissement récent, et en nombre croissant après la fin de la guerre d'indépendance américaine ${ }^{19}$. En 1791, la solution retenue fut la création de deux provinces distinctes - l'une, le Haut-Canada à majorité anglophone, située à l'ouest sur les grands lacs, et l'autre, le Bas-Canada à

\footnotetext{
${ }^{16}$ Cartwright, op. cit., p. 108.

${ }^{17}$ Ibid., p. 110.

${ }^{18}$ Ibid., p. 114.

${ }^{19}$ Voir Arthur Berriedale KEITH (ed.), Selected Speeches and Documents on British Colonial Policy 1763-1917 (1948). Pour les difficultés initiales à accommoder la population catholique dans l'Empire, voir vol. I : 'The Royal Proclamation of October 7, 1763', pp. 3-11 ; 'An Act for the Making More Effectual Provision for the Government of the Province of Quebec in North America (14 George III, c. 83)' (Quebec Act de 1774), p. 53-65 ; et 'Petition for the Repeal of the Quebec Act' (1774), pp. 66-69.
} 
majorité francophone, sur les rives du Saint-Laurent - chacune dotée de conseils législatif et exécutif ainsi que d'une assemblée représentative.

À la fin de 1837 éclate dans le Haut et le Bas-Canada une rébellion qui a pour origine une revendication insatisfaite de responsabilité de l'exécutif colonial devant la chambre d'assemblée et de réforme du conseil législatif. Dans chacune de ces provinces, ce sont en effet les gouverneurs et leurs conseils nommés qui ont la haute main sur la législation. Non seulement cela déplaît-il aux chambres élues, mais cela paraît institutionnaliser des clivages impopulaires - dans le Haut-Canada, entre l'Église d'Angleterre, seule bénéficiaire de la dîme universellement prélevée, et la majorité non anglicane des habitants ; au Bas-Canada, entre la population francophone représentée à la chambre et la minorité anglophone qui domine le conseil législatif. Une fois réprimé ce mouvement de rébellion, la question du gouvernement responsable est au cœur de la révision entreprise par le gouvernement britannique de sa politique coloniale en Amérique du nord. Dans l'exacte mesure où il craint que rendre les conseils législatifs électifs, et les gouvernements coloniaux comptables de leurs politiques devant les chambres d'assemblée, ne mène à la séparation des colonies de la métropole, l'union des deux Canada lui apparaît comme un artifice nécessaire pour préserver l'intégrité de l'Empire. En 1840, ces deux provinces sont réunies au sein d'une union législative.

Rendre au Bas-Canada l'exécutif comptable de ses actes devant la législature, croit-on par exemple, hâterait la sécession de la métropole, non seulement en vertu d'une indépendance reconnue en fait, sinon en droit, mais aussi parce que les anglophones de la province pourraient chercher alors l'appui des États-Unis. Une union législative, en absorbant l'élément français dans un plus grand tout, rendrait à l'inverse plus supportable l'existence d'un conseil législatif nommé ${ }^{20}$. Comme l'exprimera Lord John Russell devant la Chambre des communes en 1838, un conseil électif mettrait le gouvernement colonial à la merci «d'un ou deux partis extrémistes » et «de la résistance de l'autre parti naîtrait inévitablement le danger de guerre civile ». En important dans la chambre haute de la législature les passions et les idées révolutionnaires de l'assemblée, une telle réforme ne pourrait être interprétée par la minorité britannique que comme un abandon de leurs intérêts et une incitation à prendre leur avenir en main ${ }^{21}$. En outre, une telle mesure au BasCanada enclaverait totalement la province supérieure et la couperait de tout contact avec la métropole de l'Empire ${ }^{22}$. Enfin, le principe du gouvernement responsable menace d'ébranler l'édifice tout entier de l'Empire. Pour Russell, qui deviendra secrétaire aux Colonies en août 1839 ,

[...] il n' [est] qu'un lieu où le pouvoir de l'exécutif [puisse] être ainsi parfaitement discipliné (controlled), et c'est le siège du gouvernement impérial. Si le souverain de ce pays devait choisir ceux qui

\footnotetext{
${ }^{20}$ Peter BURROUGHS, The Canadian Crisis and British Colonial Policy, 1828-1841, London: Edward Arnold, 1972, p. 79.

${ }^{21}$ Lord John RUSSELL, discours du 16 janvier 1838, Hansard's Parliamentary Debates: Third Series; commencing with the accession of William IV, vol. XL, London: Thomas Curson Hansard, 1838, p. 26.

${ }^{22}$ Edward Ellice à Lord John Russell, 7 janvier 1838, cité in : BURROUGHS, op. cit., p. 4.
} 
posséderaient la confiance de la Couronne, mais nullement la confiance de la Chambre des communes, il en résulterait un prompt changement d'administration, et la constitution ne pourrait continuer de fonctionner qu'au prix de ce changement. Mais dans une colonie, si le conseil législatif doit être nommé conformément aux væux de l'assemblée, il est une autre question qui survient, à savoir, qu'advient-il des ordres donnés par le gouvernement impérial et le gouverneur de la colonie ? L'on est ici placé dans la situation où son autorité est complètement bafouée. L'on a un ministère canadien responsable devant la seule assemblée, doué du pouvoir de renvoyer ou de chasser de tel ou tel endroit les troupes britanniques et d'admettre les vaisseaux d'une puissance, peut-être en guerre avec notre pays, dans les ports du Canada. Si [ce ministère canadien] n'a pas ce pouvoir d'imposer de telles restrictions, il faut le rendre responsable. S'il l'a, alors un gouverneur suprême et indépendant n'a pas sa place aux colonies. ${ }^{23}$

Sous un tel régime, il n'y aurait plus à proprement parler de colonie au BasCanada, mais en ses lieu et place, «une république nouvelle et indépendante ${ }^{24}$. Comment, dès lors, concéder le principe de la responsabilité, quand son prix serait d'abandonner la maîtrise du Saint-Laurent aux États-Unis et de laisser s'établir à Québec une république canadienne, dont l'existence même rendrait impossible de conserver dans le giron impérial la Nouvelle-Écosse et le Nouveau-Brunswick, c'est-à-dire causerait la perte de toute l'Amérique du nord $?^{25}$ Le besoin immédiat de produire un règlement qui tout à la fois préserve une forme ou l'autre de gouvernement représentatif et mette un frein à l'influence des Canadiens français, rend donc une union législative des deux Canada «virtuellement inévitable ». En même temps, la rébellion française débarrasse le gouvernement britannique de tout scrupule à imposer sa solution ${ }^{26}$.

\section{Le gouvernement responsable}

Et pourtant le gouvernement responsable est institué dès 1848. «Par l'adoption de ce principe, écrit une autorité constitutionnelle du temps,

[...] une constitution coloniale devient l'image et le reflet exacts du gouvernement parlementaire de l'Angleterre. Le gouverneur, à l'instar du souverain qu'il représente, se tient à l'écart et au-dessus des partis ; et gouverne par le truchement de conseillers constitutionnels qui disposent d'un ascendant dans la législature. Il laisse les partis en

\footnotetext{
${ }^{23}$ Lord John RUSSELL, discours à la Chambre des communes, 16 janvier 1838, loc. cit.

${ }^{24}$ Ibid., p. 27.

${ }^{25}$ Hansard's Parliamentary Debates: Third Series; commencing with the accession of William IV, Vol. XL, op. cit., p. 34.

${ }^{26}$ Ged W. MARTIN, 'The influence of the Durham Report', in : Ronald HYAM \& Ged MARTIN (eds.), Reappraisals in British Imperial History, London \& Basingstoke: Macmillan, 1975, pp. 33 \& 37 (citation à la p. 33) ; et du même auteur, 'Confederation Rejected: The British Debate on Canada, 1837-1840', Journal of Imperial and Commonwealth History, vol. XI, n 1 (October 1982), pp. 33-57 (pp. 78, 80).
} 
rivalité se livrer bataille jusqu'au bout ; et en admettant le parti le plus fort dans ses conseils met l'autorité de l'exécutif en harmonie avec les sentiments populaires. ${ }^{27}$

Son introduction intervient à un moment où le principe de la responsabilité est encore mal établi au Royaume-Uni. Avec l'élargissement du suffrage en 1832, en effet, les majorités ne sont plus acquises par le biais du contrôle exercé par la monarchie et les grandes familles, tandis que le système moderne des partis n'en est qu'à ses balbutiements et n'assure pas encore de discipline stricte (le parti tory se divise en 1846 sur la question de l'abrogation des lois céréalières). C'est le signe que c'est tout autant le pragmatisme et les circonstances politiques qu'un principe constitutionnel, clair en théorie mais incertain en pratique, qui dictent la mesure. L'union de 1840, et les déclarations qui la précèdent ou l'accompagnent, le démontrent suffisamment.

La Nouvelle-Écosse et le Canada uni en sont les premiers bénéficiaires. Là, le vieux système de gouvernement représentatif sans consentement rendait l'exécutif prisonnier des animosités et querelles entre partis (au Canada, entre anglophones et francophones ; en Nouvelle-Écosse entre loyalistes et libéraux), dépendant tantôt des uns, tantôt des autres pour pouvoir gouverner. Cela affaiblissait son action et rendait la colonie politiquement instable. Et lorsque Londres a concédé le principe, il est ensuite étendu aux autres provinces maritimes d'Amérique du nord, puis aux autres colonies de peuplement, même lorsqu'elles ne possèdent pas le personnel politique et l'organisation partisane qui sont les conditions de son bon fonctionnement - ce qui rend parfois difficile (comme, par exemple, au Nouveau-Brunswick) de déterminer quand le gouvernement responsable y est véritablement introduit. Sur le continent australien, l'Australie méridionale et la Nouvelle Galles du Sud y accèdent dans les années 1850 ; la colonie de Victoria en 1855 (cinq ans après sa séparation de la Nouvelle Galles du Sud) ; le Queensland en 1859 ; l'Australie occidentale en 1890. Dans la Confédération canadienne, après les provinces maritimes, c'est le tour du Manitoba en 1870, de la Colombie britannique en 1871. Ailleurs dans l'Empire, on retiendra encore la Nouvelle-Zélande en 1856, la colonie du Cap en 1871, et celle du Natal en 1893.

L'instauration du «gouvernement responsable » dans l'empire est rendue possible par une division empirique et souple de l'activité gouvernementale entre domaines d'intérêt propre aux seules colonies et domaines qui engagent les intérêts de la Grande-Bretagne et de 1'Empire dans son ensemble ${ }^{28}$. Cette division concilie l'unité du tout et la liberté des parties. Le libre échange, sous la forme de l'abrogation des lois protectionnistes sur le blé (Corn Laws) et sur le transport maritime (Navigation Acts) respectivement en 1846 et 1849, est en passe de devenir

\footnotetext{
${ }^{27}$ Thomas Erskine MAY, Constitutional History of England since the Accession of George the Third, 1760-1860, Littleton, Colorado: Fred B. Rothman \& Co., 1986 (reprint de l'édition de Boston de 1862), p. 537-538.

${ }^{28}$ Préface de Frederick MADDEN \& David FIELDHOUSE (eds.), Select Documents on the Constitutional History of the British Empire and Commonwealth, Vol. IV, Settler SelfGovernment, 1840-1900, New York, Westport, Connecticut \& London: Greenwood Press, 1990, p. xxii.
} 
une réalité et l'on croit volontiers que les échanges commerciaux vont se substituer à la tutelle de Londres pour cimenter l'Empire. De la sorte, Londres évite que l'Empire ne mène au despotisme, aussi bien en métropole (par une extension des prérogatives de l'exécutif) qu'outre-mer (par un centralisme excessif). De même, la séparation des affaires proprement coloniales et des affaires impériales rend inutile le recours à la forme fédérale, inutile une cour suprême qui statuerait sur les limites d'exercice de l'autorité du gouvernement central et des législatures coloniales. La souveraineté parlementaire de Westminster, législature de l'Empire, et l'usage modéré du veto royal contre la législation coloniale permettent d'éviter les conflits d'intérêt entre métropole et colonies ${ }^{29}$.

Equilibre dynamique, donc, à l'instar de l'union métropolitaine. Et dévolution, si l'on veut, puisqu'il y a coexistence de la souveraineté parlementaire, de l'autonomie législative partielle des colonies, et de la responsabilité de l'exécutif colonial devant sa législature - ce dernier facteur important pour illustrer un parallèle avec la situation actuelle au Royaume-Uni bien plus recevable qu'une hâtive référence à Burke, qui négligerait tout ensemble la situation de l'Irlande hors de l'Union et la disjonction dans les colonies entre pouvoirs exécutif (émanation directe et active de la métropole) et législatif (non représentatif au Québec, puis dans les deux Canada).

\section{L'autonomisation de l'Empire}

En 1865, le Colonial Laws Validity Act accorde aux colonies à gouvernement responsable le droit d'amender leurs constitutions, tout en réservant par ailleurs la possibilité pour le Parlement de Westminster de légiférer à l'encontre de lois coloniales. Au fil des années, leur autonomie grandit. À la veille de la Grande Guerre, ces colonies que l'on dénomme depuis 1907 Dominions, possèdent la pleine maîtrise de leurs affaires intérieures, de leurs droits de douane, de leur réglementation de l'immigration, et commandent leurs propres forces de défense, pouvant décider de la nature et du degré de leur appui militaire à l'Empire. Dans le domaine réservé des affaires étrangères, elles participent à la négociation et au bénéfice des traités commerciaux signés par le Royaume-Uni.

Après-guerre, devant les revendications coloniales d'une autonomie accrue dans le sein d'un Empire préservé, les changements s'accélèrent. En 1923, la conférence impériale, forum de discussion entre la mère-patrie et ses colonies, reconnaît comme un fait accompli l'existence de politiques étrangères séparées, tout en soulignant la nécessité d'une coordination. En 1926 la conférence, reprenant les termes du rapport de la Commission sur les Relations impériales, ou rapport Balfour, définit les Dominions comme «des communautés autonomes au sein de l'Empire britannique, égales en statut, en aucun cas subordonnées l'une à l'autre en aucun aspect de leurs affaires intérieures ou extérieures, bien qu'unies par une commune allégeance à la Couronne, et librement associées en leur qualité de membres du Commonwealth britannique des nations ». Elle énonce solennellement le principe

${ }^{29}$ Sur la séparation des affaires coloniales et impériales, voir par exemple le discours de Sir William Molesworth, 6 mai 1850, à propos de l'Australian Colonies Government Bill, ibid., pp. $9-12$. 
selon lequel le Parlement de Westminster ne légiférera pas pour un Dominion sans son consentement.

Le contexte est parlant. En Afrique du Sud, le général Smuts - premier ministre de l'Union sud-africaine de 1919 à 1924, puis de 1938 à 1949, et l'un des artisans du Commonwealth - vient en 1920 et 1921 de repousser la menace d'un nationalisme afrikaner sécessionniste en arguant de ce que le pays ne se trouvait plus dans une position subalterne. Hertzog, son successeur en 1924, est à l'inverse le champion de l'identité afrikaner et revendique pour son pays le droit de sortir de l'Empire. La déclaration de 1926 permet donc à l'Afrique du Sud - tout comme à l'Irlande, qui s'est vu accorder le statut de Dominion en 1921, mais où le nationalisme se partage entre ceux qui acceptent cette solution et ceux qui demandent l'indépendance - de trouver un équilibre interne délicat entre l'unité impériale et une rhétorique d'indépendance nationale.

Cinq ans plus tard, le Statut de Westminster donne force de loi au rapport Balfour. Il déclare solennellement les Parlements du Canada, de l'Australie, de la Nouvelle-Zélande, de l'Union sud-africaine, de l'État libre d'Irlande et de Terre-neuve libres de toute emprise du Parlement du Royaume-Uni. En outre, la législation du Parlement de Westminster ne doit désormais s'étendre aux Dominions que sur leur demande et avec leur consentement. Le Colonial Validity Act, obsolète, est abrogé.

Il convient d'observer que le Statut ne vise nullement à préparer l'indépendance des Dominions. Au contraire, le lien impérial est pour les Dominions où la tentation sécessionniste est la plus forte, en Irlande et en Afrique du Sud, le meilleur moyen de préserver l'unité nationale. L'indépendance, au contraire, y précipiterait la division ${ }^{30}$. Le Statut de Westminster formalise une solution d'équilibre entre les aspirations nationales et l'unité de l'Empire britannique, entre l'autonomie et l'accès à l'influence internationale et à la sécurité procurée par l'association à l'une des plus grandes puissances du monde. En accomplissant la dévolution la plus complète des pouvoirs du Parlement impérial, il ressoude l'Empire de peuplement.

\section{Conclusion}

La dévolution constitue, à n'en pas douter, le changement le plus profond apporté à l'architecture constitutionnelle du Royaume-Uni depuis sa formation en 1707. Aurait-elle existé antérieurement dans le cadre plus large de l'Empire britannique ? Et si c'est le cas, pourquoi une solution pratiquée par Londres dans certaines régions de ses domaines ultramarins ne fut-elle appliquée qu'avec un si grand décalage et une si grande réticence en métropole ?

Pour ce qui a trait à la première question, nous avons vu qu'elle est en un sens mal posée. L'Empire, en effet, est extérieur à l'Union. Il représente en soi une solution à la question de l'unité dans la diversité, autre que l'union législative avec la métropole. D'autre part, l'autonomie de gouvernement que l'on y observe n'a pas

${ }^{30}$ Ce n'est pas un hasard si le Statut de Westminster est promptement adopté à Dublin et à Pretoria, quand l'Australie et la Nouvelle-Zélande, par exemple, attendent respectivement 1942 et 1947 pour l'incorporer dans leur droit municipal. 
pour fonction de maintenir l'union métropolitaine. Y rechercher un modèle de fonctionnement applicable à la métropole, où la solution retenue au même problème est précisément l'union, doit par voie de conséquence paraître hasardeux. Cela ne signifie naturellement pas que coloniaux et métropolitains ne s'y soient pas essayés. $\mathrm{Au}$ contraire, pétris de la même culture constitutionnelle, ils n'ont cessé de considérer la fascinante anomalie des rapports, au demeurant si durables, entre Union et Empire. En revanche, cela veut dire que la similitude ne saurait être parfaite.

Nous avons vu, en outre, que la comparaison est nettement plus probante pour le XIX ${ }^{\mathrm{e}}$ siècle que pour le siècle précédent. Il est alors notable qu'elle est elle-même une solution graduelle et tardive à l'échelle de l'histoire impériale. Et surtout qu'elle a pour fonction de maintenir l'unité impériale. Poser que le délitement ou la désintégration de l'Empire par le gouvernement responsable entraînerait logiquement la reproduction du phénomène en métropole sous le régime de la dévolution, ce serait donc d'abord se méprendre sur la fonction de la première de ces deux mutations constitutionnelles. Mais, plus grave, ce serait croire ou donner à croire qu'Union et Empire sont pleinement intégrés par une même constitution, ce qui est à l'évidence faux, puisque le principe de la souveraineté parlementaire commande leur séparation, et que, pour prendre une image, la dérive continentale des colonies n'affecte nullement l'union métropolitaine. L'Empire était extérieur à l'Union. Sa perte n'a dissous l'Union que dans l'esprit de ceux qui ne perçoivent pas la solution de continuité de l'un à l'autre, pourtant amplement démontrée par la crise constitutionnelle américaine, et par les difficultés insurmontables à fédéraliser l'Empire au XVIII ${ }^{\mathrm{e}}$ comme au XIX ${ }^{\mathrm{e}}$ siècle.

Quant à la seconde question, la réponse, on l'aura compris, est à chercher dans l'Union elle-même. La concession de l'autonomie, déjà si difficile dans le cadre colonial, ne pouvait que poser problème au Royaume-Uni lui-même. Mais, là encore, il n'est pas vain de relever qu'elle y soulève des craintes très semblables et qu'elle a vocation, aux yeux de ses promoteurs, à assurer l'unité.

Le parallèle le plus sûr et le plus éclairant entre Union et Empire, toutefois, réside probablement dans un fait important, mais trop souvent négligé : la capacité de la périphérie à modeler, sinon le centre, du moins sa relation au centre. Si la dévolution a tant attendu en métropole, c'est d'abord parce que la demande y était absente ou très minoritaire. Or dans l'un et l'autre cas de l'Union et de l'Empire, on constate, dès lors que la demande est formulée avec toute la netteté d'un fort soutien populaire, une même volonté de maintenir par la dévolution une unité décidée en dernier ressort (comme l'Irlande, après les États-Unis, en fournit un exemple éclatant) par la périphérie ${ }^{31}$.

\footnotetext{
${ }^{31}$ Voir Paul WARD, Unionism in the United Kingdom, 1918-1974, Basingstoke: Palgrave Macmillan, 2005, p. 186.
} 